Einführung in das Schwerpunktthema

\title{
Kooperative Umwelt-Governance am Beispiel Integrierte Produktpolitik
}

I

Von Frieder Rubik und Dirk Scheer $\mathrm{n}$ den letzten 20 Jahren hat eine allgemeine politische Blickwende hin zu Prinzipien gesellschaftlicher Selbststeuerung stattgefunden. Von Beyme beschreibt dies als Übergang von einer zentralen Steuerung zu einer Selbststeuerung, die auf eine Kooperation mit und zwischen den (zivil-) gesellschaftlichen Akteuren zielt (1). Dieses Kernelement des Zugangs zu gesellschaftlichen Problemlösungen gründet sich darin, dass die Komplexität des zu steuernden und gestaltenden Politikfeldes die Steuerungsfähigkeit des Staates vielfach überfordert.

Eines der Kernprinzipien der Umweltpolitik, zumindest in Deutschland, ist das Kooperationsprinzip. Mehr als Leitlinie in den siebziger Jahren entstanden, fand es einen stärkeren Eingang in die Umweltpolitik erst zwanzig Jahre später. Heute scheint es kaum noch wegzudenken zu sein. Allerdings zeigen Beispiele der letzten Jahre, wie etwa die freiwillige Zusage der deutschen Industrie zur Reduktion von Kohlendioxid oder die Zusage zur Einhaltung bestimmter Mehrwegquoten, deutliche Implementations- und Diffusionsdefizite: Kooperative Lösungen, die ordnungsrechtliche Maßnahmen substituieren sollen, scheinen an Glaubwürdigkeit verloren zu haben und ein „Roll-back“ interventionistischer Konzepte zu begünstigen.

Dieses Spannungsverhältnis steht im Blickpunkt dieser Schwerpunktausgabe. Dabei wird beispielhaft auf Integrierte Produktpolitik abgezielt. Integrierte Produktpolitik (IPP) ,setzt an Produkten und Dienstleistungen und deren ökologischen Eigenschaften während des gesamten Lebensweges an; sie zielt auf die Verbesserungen ihrer ökologischen Eigenschaften ab und fördert hierzu Innovationen von Produkten und Dienstleistungen (2). IPP soll in diesem Heft beispielhaft für eine neue Form des Regierens stehen. Im Mittelpunkt steht dabei die Debatte um einen ganzheitlichen versus einen problembezogenen ,hot-spot“ - Ansatz und um die Rollen politischer Akteure.

\section{- Die Beiträge im Überblick}

Chris van Rossem, Beatrice Kogg und Oksana Mont eröffnen das Schwerpunktthema und geben einen kritischen Überblick über die Strategien der EU-Kommission zu einer Integrierten Produktpolitik. Sie kritisieren insbesondere die ungeklärten Rollen von Staat (EU und Mitgliedsstaaten), Wirtschaft und zivilgesellschaftlichen Akteuren in der im vergangenen Jahr vorgelegten IPP-Mitteilung der Kommission. Sie sehen es als notwendig an, dass eine politische Entscheidung über unterschiedliche Entwicklungsrichtungen einer IPP getroffen wird, um damit einen Pfad mit einer klaren Zielrichtung einzuschlagen, der anderen Akteuren verlässliche Signale sendet.

Danach interpretieren Dirk Scheer und Frieder Rubik Integrierte Produktpolitik als ein Beispiel für strukturell bedingtes Staatsversagen. Sie betrachten IPP als beispielhaft für einen Paradigmenwechsel von Ordnungsrecht zu gesellschaftlicher Selbststeuerung. Wie allerdings gesellschaftliche Selbststeuerung gestalten? Die Autoren entwickeln dabei mehrere Ansatzpunkte für produktpolitische Kooperationsstrategien, die sich an den Politikphasen der Problemwahrnehmung, Politikformulierung sowie Politikimplementation orientieren.

Erfahrungen mit einem derartigen kooperativen Ansatz führt Siv Näslund auf. Sie berichtet von zwei schwedischen Dialogforen, die auf Veranlassung der schwedischen Regierung durchgeführt wurden. In den beiden Bereichen - Bauen und Wohnen sowie Lebensmittelhandel - wurden Akteure zusammengebracht, die gemeinsam innovative und von einer Vision für das Jahr 2025 abgeleitete Maßnahmen vereinbarten und freiwillig umsetzen.

Die Bekanntheit und das Verständnis einer IPP gerade in der Wirtschaft stellen Siegfried Kreibe und Michael Schneider vor. Ausgehend von zwei repräsentativen Umfragen in der bayerischen produzierenden Industrie zeigt sich ein bescheidener Kenntnisstand zu IPP. Bemerkenswert ist der Zweifel der Mehrheit der Befragten an einem ausschließlichen Ansatz über Selbstverpflichtungen; vielmehr wird hier durchaus eine Rolle für die Politik gesehen.

Ausgehend vom Beispiel des gegenwärtig in der EU diskutierten Kommissionsvorschlags zu Energie verbrauchenden Produkten sieht Christian Hey eine Auseinandersetzung zwischen zwei IPP-
Modellen: Einem ganzheitlichen Ansatz, der auf verbandlicher Selbststeuerung beruht, und einem fokussierten Ansatz, der ambitionierte Effizienzstandards für den Energieverbrauch anstrebt. Er interpretiert diese Modelle als eine Auseinandersetzung zwischen einem status-quo-bezogenen und einem innovations- und effizienzanspornenden regulativen Ansatz.

Der japanische Toprunneransatz, der seit einigen Monaten in der umweltpolitischen Diskussion für Furore sorgt, wird von Heike Schröder vorgestellt. Dieses Konzept fokussiert auf den Energieverbrauch von rund 20 verschiedenen Produktkategorien und hat eine ordnungsrechtliche Zielkomponente.

Der Beitrag von Klaus Jacob und Gesine Foltjanty-Jost richtet schließlich den Blick auf ein bereits etabliertes Politikfeld - und ihre Kooperationsstrukturen. Am Beispiel der deutschen Klimapolitik weisen die Autoren ein kooperatives und offenes klimapolitisches Netzwerk nach. Dabei ist interessant, dass trotz unterschiedlicher Ressourcenausstattung und Formen der Einflussnahme bei den Akteuren dies offensichtlich eine Kultur der Kooperation nicht behindert.

Insgesamt zeigen die verschiedenen Beiträge beispielhaft die Breite der Debatte um eine kooperative Herangehensweise in der Umweltpolitik auf, fokussiert auf das „Ergrünen“ von Produkten und Dienstleistungen; ein zukïnftig erfolgreicher Weg einer Integrierten Produktpolitik wird nicht ausschließlich in marktlicher Selbststeuerung, sondern eher zugunsten eines Governance-Mixes aus Kooperation und Intervention gesehen.

\section{Anmerkungen:}

(1) von Beyme, Klaus: Theorie der Politik im 20. Jahrhundert. Von der Moderne zur Postmoderne. Frankfurt a.M 1991

(2) Rubik, Frieder: Integrierte Produktpolitik. Marburg 2002, S. 17.

\section{Die Autoren:}

Dirk Scheer ist wissenschaftlicher Mitarbeiter am, Dr. Frieder Rubik Leiter des Forschungsfelds Ökologische Produktpolitik des Instituts für ökologische Wirtschaftsforschung (IÖW).

Kontakt: IÖW (Büro Heidelberg), Bergstr. 7, 69120 Heidelberg. Tel. 06221-649163, Fax 06221-27060, E-Mail: dirk.scheer@heidelberg.ioew.de, frieder.rubik@ioew.de 
(c) 20I0 Authors; licensee IÖW and oekom verlag. This is an article distributed under the terms of the Creative Commons Attribution Non-Commercial No Derivates License (http://creativecommons.org/licenses/by-nc-nd/3.o/), which permits unrestricted use, distribution, and reproduction in any medium, provided the original work is properly cited. 